\title{
Le microcrédit personnel : un modèle au potentiel inclusif
} limité

\author{
Personal microcredit: A model with limited potential for \\ inclusion
}

\section{El microcrédito: un modelo con un potencial integrador limitado}

\section{Jean-Marc Figuet et Fabienne Pinos}

Numéro 335, janvier 2015

URI : https://id.erudit.org/iderudit/1028535ar

DOI : https://doi.org/10.7202/1028535ar

Aller au sommaire du numéro

Éditeur(s)

Association Recma

ISSN

1626-1682 (imprimé)

2261-2599 (numérique)

Découvrir la revue

Citer cet article

Figuet, J.-M. \& Pinos, F. (2015). Le microcrédit personnel : un modèle au potentiel inclusif limité. Revue internationale de l'économie sociale, (335), 87-101. https://doi.org/10.7202/1028535ar
Résumé de l'article

Cet article a pour objet de dresser un état des lieux de l'utilisation du microcrédit personnel garanti par le Fonds de cohésion sociale (FCS ; loi ${ }^{\circ}$ 2005-32 du 18 janvier 2005 de programmation pour la cohésion sociale) depuis sa création en France, en 2005. Il montre que des freins matériels et conceptuels inhérents aux caractéristiques du dispositif en limitent l'usage et l'efficacité. A partir de données chiffrées, il met en évidence que les coûts et les contraintes organisationnelles assumés par les établissements financiers prêteurs et les intermédiaires sociaux condamnent une distribution plus massive du microcrédit personnel. Il souligne, ensuite, qu'en dépit d'études d'impacts optimistes, menées par exemple par la Fédération nationale des Caisses d'épargne (2010) ou plus récemment à la demande de la Caisse des dépôts et consignations (Gloukoviezoff, Rebière, 2013b), la conception même du dispositif peut conduire à des dépenses difficilement justifiables pour l'Etat. Il conclue sur la nécessaire rénovation du dispositif en profondeur pour en faire un réel facteur d'inclusion. 


\title{
LE MICROCRÉDIT PERSONNEL: UN MODĖLE AU POTENTIEL INCLUSIF LIMITÉ
}

\author{
par Jean-Marc Figuet* et Fabienne Pinos**
}

\footnotetext{
* Larefi, université de Bordeaux. Mél. : jean-marc. figuet@u-bordeaux.fr.

** Larefi, université de Bordeaux. Mél. : fabienne. pinos@u-bordeaux.fr.
}

Cet article a pour objet de dresser un état des lieux de l'utilisation du microcrédit personnel garanti par le Fonds de cohésion sociale (FCS; loi $n^{\circ} 2005-32$ du 18 janvier 2005 de programmation pour la cohésion sociale) depuis sa création en France, en 2005. Il montre que des freins matériels et conceptuels inhérents aux caractéristiques du dispositifen limitent l'usage et l'efficacité. A partir de données chiffrées, il met en évidence que les coûts et les contraintes organisationnelles assumés par les établissements financiers prêteurs et les intermédiaires sociaux condamnent une distribution plus massive du microcrédit personnel. Il souligne, ensuite, qu'en dépit d'études d'impacts optimistes, menées par exemple par la Fédération nationale des Caisses d'épargne (2010) ou plus récemment à la demande de la Caisse des dépôts et consignations (Gloukoviezoff, Rebière, 2013b), la conception même du dispositif peut conduire à des dépenses difficilement justifiables pour l'Etat. Il conclue sur la nécessaire rénovation du dispositifen profondeur pour en faire un réel facteur d'inclusion.

\section{Personal microcredit: A model with limited potential for inclusion}

This article assesses the use of personal microcredit guaranteed by the Social Cohesion Fund since its creation in France, in 2005. It shows that material and conceptual limitations inherent to the scheme hamper its use and effectiveness. The data highlight that the costs and organisational constraints assumed by lending financial institutions and social intermediaries weigh heavily against a more massive expansion of personal microcredit. Despite the optimistic impact reports undertaken by the Fédération nationale des Caisses d'épargne (2010), for example, or more recently at the request of the Caisse des dépôts et consignations (Gloukoviezoff, Rebière, $2013 b$ ), the article emphasizes that the scheme's actual design can lead to expenditures that are hard for the government to justify. The article concludes on the need for a complete overhaul of the scheme to make it a real driver of inclusion.

\section{El microcrédito: un modelo con un potencial integrador limitado}

El presente artículo tiene como objetivo establecer un estado de la utilización del microcrédito personal garantizado por el Fondo de cohesión social desde su creación en Francia, en 2005. El estudio muestra que algunos obstáculos materiales y conceptuales, inherentes a las características del dispositivo, limitan el uso y la eficiencia del mismo. A partir de datos cuantificados, pone en evidencia que los costos y las restricciones de carácter organizacional asumidas por los establecimientos financieros prestadores y los intermediaros sociales impiden una distribución masiva del microcrédito personal. Luego, el articulo destaca que a pesar de los estudios de impacto optimistas, realizados por ejemplo por la Fédération nationale des Caisses d'épargne (2010), 
o más recientemente a petición de la Caisse des dépôts et consignations (Gloukoviezoff, Rebière, 2013b), la concepción misma del dispositivo podría acarrear gastos difícilmente justificables desde la mirada del Estado. Concluye sobre la necesaria renovación del dispositivo para que sea un verdadero factor de integración.

Environ dix ans après les premiers microcrédits professionnels, la loi de cohésion sociale, dite aussi plan Borloo, donne naissance en 2005 aux microcrédits personnels (MCP) garantis par le Fonds de cohésion sociale (FCS). Ce dispositif ne répond pas à une situation de rationnement global du crédit, puisque l'on constate au début des années 2000 que «l'évolution des offres tend vers la promotion de l'endettement perpétuel pour tous» (Ducourant, 2010, p. 47).

Dès son origine, le microcrédit personnel s'apparente à une action de politique sociale qui doit permettre le financement de "projets de vie » dans des conditions soutenables pour un public défavorisé. Selon Glémain et Meyer (2011, p. 275), «le microcrédit personnel garanti s'inscrit dans [1e] renouveau de l'action sociale». En le soutenant, les pouvoirs publics reconnaissent l'existence en France de foyers aux conditions de ressources insuffisantes pour accéder au financement d'un projet de vie émancipateur.

Les microcrédits personnels se révèlent des dispositifs hybrides: ils poursuivent des objectifs sociaux, mais conservent "toutes les caractéristiques du crédit tel qu'il se développe dans les organisations (coopératives et commerciales) bancaires depuis la fin du XIX ${ }^{e}$ siècle » et s'inscrivent "dans un modèle d'économie de marché» (Glémain, Moulévrier, 2011). L’Etat recherche dans la sphère privée des supports et des méthodes capables de pallier des insuffisances constatées dans les politiques sociales. Ce constat introduit le paradoxe que son modèle économique doit dépasser: celui de l'équilibre financier et de la pérennisation de l'action solidaire.

Entre 2006 et 2012, 41214 microcrédits personnels garantis à $50 \%$ par la Caisse des dépôts et consignations (CDC) ont été distribués. Confidentielle en 2006 avec 457 microcrédits octroyés, la distribution a connu une forte accélération par la suite. Ainsi, en 2012, 11870 MCP ont été souscrits. Les contributions inégales des réseaux bancaires et de certaines sociétés de crédit à la consommation ont permis de cumuler, entre 2006 et 2012, un montant total de prêts élevé à 92 millions d'euros, soit en moyenne 2232 euros par prêt. En comparaison, l'encours total des crédits à la consommation aux particuliers en France s'élevait à 147 milliards d'euros en décembre $2012^{(1)}$. Cet écart d'échelle montre le caractère encore expérimental du microcrédit personnel. Peu d'études se sont attachées à estimer les besoins auxquels ce dispositif pourrait correspondre sur le territoire français. Les évaluations existantes convergent vers "le chiffre de 490000 emprunteurs potentiels " (Gloukoviezoff, Rebière, 2013a). Ainsi, en plus de six ans d'existence, ce dispositif aurait touché moins de $10 \%$ du public pour lequel il a été conçu. Ce constat amène à s'interroger sur les freins à la diffusion plus massive du MCP. Nous montrons que ceux-ci sont à la fois matériels et conceptuels et indissociables des caractéristiques actuelles du dispositif soutenu par l'Etat ${ }^{(2)}$.

(1) Banque de France (2013), www.banque-france.fr/uploads/ tx_bdfstatistiquescalendrier/ 2013-03-stat-info-credit-ala-consommation.pdf.

(2) D'autres offres de microprêts peuvent exister localement, telles que le prêt stabilité du Crédit municipal de Nantes (Glémain, dir., 2012). 
L'étude des publications réalisées par les différents acteurs financiers du MCP permet de détecter une similitude notable. Certes, tous communiquent sur leur participation au dispositif, affichent le nombre de prêts décaissés, parfois le nombre de collaborateurs impliqués, voire une évaluation des effets sur l'emploi des emprunteurs, mais aucun ne publie d'analyse chiffrée approfondie qui pourrait mettre en évidence les impacts économiques des MCP sur les emprunteurs, sur les associations conventionnées ou sur les établissements prêteurs eux-mêmes. L'absence d'étude mettant en relation, avec précision, moyens engagés et résultats obtenus interroge sur l'existence d'une véritable stratégie de développement de ce dispositif dans les établissements financiers, qui serait distincte d'une stratégie de diversion de l'opinion publique. Celle-ci serait invitée à porter son regard sur la participation au dispositif, valorisante donc largement mise en avant, au détriment des effets et des conditions de sa réalisation, passés sous silence.

Cet article a pour objet d'étudier les freins majeurs à la diffusion du dispositif dans sa forme actuelle. Nous montrons, dans une première partie, que les coûts et les contraintes organisationnelles assumés par les prêteurs et les intermédiaires sociaux condamnent une distribution plus massive. Nous mettons en évidence, dans une deuxième partie, qu'en dépit d'études d'impacts optimistes la conception même du dispositif peut conduire à des dépenses difficilement justifiables pour l'Etat. Nous concluons sur la nécessaire rénovation du dispositif en profondeur pour en faire un réel facteur d'inclusion.

\section{Une impasse économique prévisible pour le secteur privé inhérente aux caractéristiques de «l'offre »}

(3) http://vosdroits.servicepublic.fr/particuliers/F21375. xhtml\#.

Fin 2013, le microcrédit personnel est officiellement présenté comme un crédit accompagné, d'un montant allant de 300 à 3000 euros, d'une durée de six mois à quatre ans, pour un taux, "fixépar le prêteur, généralement situé entre 1,5 et $4 \%{ }^{(3)}$. Par ailleurs, les accompagnateurs sociaux sont $82 \%$ à considérer qu'un taux inférieur à $4 \%$ est adapté et $100 \%$ à considérer qu'un taux supérieur à $6 \%$ est trop élevé (Glouviezoff, Rebière, 2013a).

Un rapide calcul montre que l'offre, telle qu'elle est conçue par les pouvoirs publics, amène à une impasse économique.

Considérons un prêt de 2500 euros accordé au taux de $4 \%$ sur trente-trois mois pour lequel le taux de refinancement de l'établissement prêteur est de $0,75 \%$ : il génère une marge d'intérêt de 117 euros. Supposons une perte moyenne de $14 \%$, soit $7 \%$ pour la banque après appel en garantie; le montant de la perte atteint en moyenne 175 euros par prêt

(4) Le «temps banque» ne correspond pas à un temps de contact avec un chargé de clientèle, mais à un temps global d'« usage » de la banque par construction supérieur au temps de contact réel. de 2500 euros accordé, soit une perte nette pour l'établissement prêteur de 58 euros, avant même de couvrir ses frais de traitement. Dans ces conditions, il faudrait appliquer un taux nominal de $7 \%$, afin que la marge d'intérêt (227,48 euros) soit supérieure au coût du risque (175 euros). Il resterait alors 52 euros pour couvrir les frais de structure, soit environ trente-cinq minutes de «temps banque » ${ }^{(4)}$, 
avec une hypothèse de coût horaire global à 87 euros ${ }^{(5)}$. Du point de vue des prêteurs, pour maintenir un taux à $4 \%$, les sinistres, après mise en jeu de la garantie CDC, devraient être inférieurs à $3 \%$ des encours prêtés, et les processus bancaires, suffisamment industrialisés pour limiter les frais de traitement des dossiers (tableau 1). Par défaut, pour atteindre un équilibre, il faudrait envisager soit une augmentation des taux nominaux, difficilement compatible avec la mission sociale véhiculée par le dispositif MCP, soit d'autres sources d'avantages, matériels ou immatériels, au bénéfice des prêteurs.

Au 31 décembre 2012, la CDC avait enregistré 216 appels en garantie depuis le démarrage du dispositif, correspondant à un montant total de dette échue impayée de 3,12 millions d'euros, soit une perte moyenne par dossier défaillant de 1408 euros. Au titre de la garantie accordée, 50 \% de ces pertes, soit 1,56 million d'euros, ont été remboursés aux prêteurs. Concernant les taux de sinistre, la CDC favorise une communication globale sur le nombre de prêts non remboursés comparativement au nombre total de prêts accordés, soit un chiffre affiché de 5,38 \%. Il y a lieu de relativiser ce pourcentage, qui peut visuellement apparaître comme une performance satisfaisante et éloignée de l'hypothèse de perte moyenne de $14 \%$ considérée supra.

Statistiquement, les pertes ne sont que très rarement constatées lors de la première année de remboursement du prêt. Les premières échéances impayées apparaissent majoritairement après douze à dix-huit mois de remboursement pour les MCP. Sur un portefeuille stabilisé ou à faible progression, ce calcul pourrait être significatif. Ici, plus de la moitié des prêts du portefeuille (53\%) ont été décaissés en 2011 (24\%) ou en 2012 (29\%). Aussi, même s'il est exact de notifier que 5,4 \% des prêts octroyés ont nécessité un appel en garantie, il serait plus significatif, si l'objectif est d'évaluer le risque, de détailler ce ratio par cohorte. D’une part, une autre approche, moins détaillée mais aussi significative, consisterait à publier en nombre et en montant la part des prêts avec appel en garantie rapportée aux prêts échus, que l'échéance soit " naturelle " - à l'issue du correct remboursement du prêt - ou « forcée » - avec passage à pertes et appel en garantie.

\section{Tableau 1}

\section{Marge d'intérêt et coût du risque}

\begin{tabular}{|r|r|r|r|r|}
\hline \multicolumn{2}{|l|}{$\begin{array}{l}\text { Prêt de } 2500 \text { euros sur trente-trois mois } \\
\text { Taux de refinancement : } \mathbf{0 , 7 5} \%\end{array}$} & \multicolumn{3}{|c|}{ Taux nominal (marge d'intérêt en euros) } \\
\cline { 2 - 5 } & Coût du risque (euros) & $\mathbf{4 1 6 , 7 6}$ & $5 \%$ & $7 \%$ \\
\hline Taux sinistre & $\mathbf{1 7 5}$ & $-58,24$ & $-21,64$ & $\mathbf{1 5 3 , 3 6}$ \\
\hline $7 \%$ & $\mathbf{1 2 5}$ & $-8,24$ & 28,36 & 102,48 \\
\hline $5 \%$ & $\mathbf{7 5}$ & 41,76 & 78,36 & 152,48 \\
\hline $3 \%$ & & & & 52,48 \\
\hline
\end{tabular}


Ces taux permettraient de mieux identifier la proportion de prêts correctement remboursés. D’autre part, il serait utile de compléter ces données par une évaluation des impayés sur l'encours brut. Le ratio affiché ne prend en effet en compte que les pertes avérées, et non les pertes prévisionnelles, alors même que plus de la moitié des prêts a été distribuée en 2011 et 2012. Sur la base des deux séries d'hypothèses de prêts échus (tableau 2), le taux de sinistralité en nombre s'établirait entre 12 et $15 \%$.

En valeur, sur la base d'un montant moyen par prêt accordé de 2232 euros, le montant initial prêté correspondant aux 2216 prêts non remboursés attendrait 4,95 millions d'euros. La perte s'élève à $63 \%$ du montant initial pour ces prêts et à 3,4\% des 92 millions d'euros prêtés depuis 2006. Les deux séries d'hypothèses appliquées à des montants moyens de prêts calculés par année amènent à évaluer un taux de sinistralité en montant compris entre 7,5 et 9,3\% (tableau 3 ).

\section{Tableau 2}

Taux de sinistralité des microcrédits personnels (en nombre, 2009-2012)

\begin{tabular}{|c|c|c|c|c|c|}
\hline \multicolumn{2}{|c|}{ Distribution } & Hypothèse 1 & Echus H1 & Hypothèse 2 & Echus H2 \\
\hline 2006 & 457 & $100 \%$ & 457 & $100 \%$ & 457 \\
\hline 2007 & 1979 & $100 \%$ & 1979 & $100 \%$ & 1979 \\
\hline 2008 & 3563 & $100 \%$ & 3563 & $100 \%$ & 3563 \\
\hline 2009 & 5520 & $100 \%$ & 5520 & $100 \%$ & 5520 \\
\hline 2010 & 7884 & $45 \%$ & 3548 & $50 \%$ & 3942 \\
\hline 2011 & 9941 & $0 \%$ & 0 & $20 \%$ & 1988 \\
\hline 2012 & 11870 & $0 \%$ & 0 & $10 \%$ & 1187 \\
\hline Total & 41214 & & 15067 & & 18636 \\
\hline \multicolumn{2}{|c|}{ Appels : 2216} & \multicolumn{2}{|c|}{ Sinistralité : 14,7\% } & \multicolumn{2}{|c|}{ Sinistralité : $11,9 \%$} \\
\hline
\end{tabular}

Source : calculs d’après les données de la CDC, journée du microcrédit LBP 2013.

\section{Tableau 3}

Taux de sinistralité des microcrédits personnels (en valeur, 2009-2012)

\begin{tabular}{|c|c|c|c|c|c|}
\hline \multicolumn{2}{|c|}{ Distribution k€ } & Hypothèse 1 & Echus H1 & Hypothèse 2 & Echus H2 \\
\hline 2006 & 1020 & $100 \%$ & 1020 & $100 \%$ & 1020 \\
\hline 2007 & 4417 & $100 \%$ & 4417 & $100 \%$ & 4417 \\
\hline 2008 & 7953 & $100 \%$ & 7953 & $100 \%$ & 7953 \\
\hline 2009 & 12321 & $100 \%$ & 12321 & $100 \%$ & 12321 \\
\hline 2010 & 17597 & $45 \%$ & 7919 & $50 \%$ & 8799 \\
\hline 2011 & 22188 & $0 \%$ & 0 & $20 \%$ & 4438 \\
\hline 2012 & 26494 & $0 \%$ & 0 & $10 \%$ & 2649 \\
\hline Total & 91990 & & 33629 & & 41596 \\
\hline \multicolumn{2}{|c|}{ Appels : 3120} & \multicolumn{2}{|c|}{ Sinistralité : 9,3\% } & \multicolumn{2}{|c|}{ Sinistralité : 7,5 \% } \\
\hline
\end{tabular}

Source : calculs d'après les données de la CDC, journée du microcrédit LBP 2013. 
Fin 2012, les 92 millions d'euros prêtés sous forme de microcrédits personnels depuis 2006 ont déjà produit 3,12 millions de pertes, soit 3,4\% de l'encours prêté - vif ou échu - et, selon nos hypothèses théoriques, plus de 7,5\% de l'encours échu.

Une analyse plus précise des échéances impayées et des pertes enregistrées a été réalisée sur un portefeuille représentatif de près de 2450 prêts distribués entre 2007 et 2012.

\section{Résultats obtenus sur l'analyse des impayés}

Au 31 décembre 2012, nous observons que 24,7\% des dossiers en cours présentent au moins une échéance impayée. Sont considérés comme dossiers en cours les prêts dont le capital restant dû (CRD) est non nul, ainsi que les prêts dont le CRD est nul mais qui ont une ou plusieurs échéances impayées. Entre 2007 et 2012, le montant total des échéances impayées s'élève à 3,34 \% du montant total décaissé pour ces prêts à l'origine. La part des créances présentant un impayé dans l'encours brut s'élève à 20,4\%, dont $10 \%$ ont enregistré plus de deux échéances impayées. Fin 2011, la Banque de France évalue à 3,13 \% le taux d'encours douteux bruts sur l'ensemble des crédits à la clientèle non financière (Autorité de contrôle prudentiel, 2012). Sans que les données soient strictement comparables, elles indiquent un risque majoré pour les encours de MCP par rapport à l'ensemble des crédits distribués à la clientèle non financière.

Près d'un tiers des prêts distribués en 2010 et 2011 présente au moins un impayé à la fin de l'année 2012. Les fréquences d'impayés sur les prêts distribués en 2008 et 2009 sont moins significatives, car ceux-ci ne constituent respectivement que $0,2 \%$ et $4,5 \%$ des 1909 prêts en cours.

La plus faible fréquence de prêts avec impayés sur 2012 montre que les risques de défaillance sont moindres la première année de remboursement. Les prêts d'un montant initial inférieur à 1000 euros représentent, en nombre, $8,7 \%$ des prêts avec impayés, mais $12,8 \%$ des dossiers. A contrario, les prêts de 3000 euros et plus pèsent pour $42,3 \%$ des prêts avec impayés, alors qu'ils ne représentent que 39,3\% des prêts distribués. Les prêts d'une durée initiale inférieure ou égale à dix-huit mois ne représentent que $6,4 \%$ des prêts avec impayés, alors qu'ils pèsent pour près de $12 \%$, en nombre, dans le portefeuille. Par ailleurs, 13,5 \% des prêts ont été accordés à des bénéficiaires fichés

\section{Tableau 4}

Microcrédits personnels, impayés sur panel

\begin{tabular}{|l|c|c|c|c|c|c|}
\hline $\begin{array}{l}\text { Année de décaissement } \\
\text { du prêt }\end{array}$ & $\mathbf{2 0 0 7}$ & $\mathbf{2 0 0 8}$ & $\mathbf{2 0 0 9}$ & $\mathbf{2 0 1 0}$ & $\mathbf{2 0 1 1}$ & $\mathbf{2 0 1 2}$ \\
\hline $\begin{array}{l}\text { \% de dossiers avec impayés (nombre } \\
\text { de dossiers en défaut par le total } \\
\text { des dossiers) [prêts en cours] }\end{array}$ & - & $25 \%$ & $38,4 \%$ & $30,9 \%$ & $31 \%$ & $16,8 \%$ \\
\hline $\begin{array}{l}\text { \% impayés sur prêts en cours } \\
\text { (impayés par le montant initial } \\
\text { décaissé) [prêts en cours] }\end{array}$ & - & $0,5 \%$ & $3,8 \%$ & $4,5 \%$ & $5,3 \%$ & $1,4 \%$ \\
\hline
\end{tabular}

Source: calculs après recueil d'informations auprès des prêteurs. 
(fichier central des chèques, FCC; fichier national des incidents de remboursement de crédit aux particuliers, FICP); ceux-ci sont 20,8 \% parmi les débiteurs avec au moins une échéance impayée. En outre, $35,8 \%$ des prêts ont été accordés à des hommes; ceux-ci sont 43,5\% parmi les débiteurs avec au moins une échéance impayée.

Nous pouvons en déduire que le montant du prêt accordé conditionne les risques de défaillance: ceux-ci sont moins présents pour les prêts inférieurs à 1000 euros, plus fréquents sur les prêts de 3000 euros et plus. De même, une durée courte réduit les risques de défaillance. A contrario, ces derniers sont majorés pour un prêt accordé à une personne fichée à la Banque de France (FCC ou FICP) ou de sexe masculin.

Concernant le critère du risque, ces statistiques plaident en faveur d'une orientation de la distribution vers des prêts de très faibles montants sur des durées très courtes. Cependant, compte tenu du mode de distribution actuel, les coûts administratifs de ces prêts sont équivalents à ceux d'un montant plus élevé, alors que le PNB généré est moindre. Leur faible diffusion $(12,8 \%$ des dossiers) pourrait être imputable à une réticence des offreurs plus qu'à un manque d'intérêt des emprunteurs. De même, ces statistiques pourraient conduire à favoriser les prêts aux femmes et aux personnes non fichées. Cette sélection amènerait à reproduire les modèles bancaires et irait à l'encontre de l'approche centrée sur le "devenir du bénéficiaire » préconisée pour le microcrédit personnel (IGF, 2010).

\section{Résultats obtenus sur l'analyse des pertes (appels en garantie)}

Au 31 décembre 2012, 14,7\% des dossiers échus, que l'échéance soit " naturelle » - à l'issue du correct remboursement du prêt - ou «forcée » - avec passage à pertes et appel en garantie -, ont fait l'objet d'un appel en garantie. Les pertes sur prêts échus se sont élevées à $14,2 \%$ des montants prêtés, également répartis entre le distributeur et la CDC.

L'analyse montre que les prêts d'un montant initial de 2000 à 2999 euros ou accordés à des personnes de sexe masculin font plus fréquemment l'objet d'un appel en garantie que la moyenne du portefeuille. Une plus forte concentration du risque est également constatée chez certains réseaux partenaires: trois d'entre eux représentent $24,7 \%$ des prêts accordés, mais $35,5 \%$ des prêts avec appel en garantie. L'évaluation théorique issue d'hypothèses de prêts échus et l'analyse d'un portefeuille représentatif de MCP garantis nous permettent d'approcher un taux de pertes plus significatif que l'affichage CDC biaisé par la forte progression de la production en 2011 et 2012 (tableau 5).

\section{Tableau 5}

Taux de perte

\begin{tabular}{|l|c|c|c|c|}
\hline Taux de perte & Affiché CDC & Hypothèses 1 & Hypothèses 2 & Réel observé \\
\hline En nombre & $5,8 \%$ & $14,7 \%$ & $11,9 \%$ & $14,7 \%$ \\
\hline En montant & $3,4 \%$ & $9,3 \%$ & $7,5 \%$ & $14,2 \%$ \\
\hline
\end{tabular}

Source : calculs après recueil d'informations auprès des prêteurs et de la CDC. 
Les modalités de calcul proposées par la CDC affichent pour le MCP des taux de sinistralité significativement en deçà des taux réels observés sur un panel représentatif de crédits.

En conclusion, du point de vue du prêteur, les conditions moyennes constatées en 2012 n'engagent pas sur un simple argument économique à développer le microcrédit personnel garanti. La faible marge d'intérêt dégagée ne couvre pas le coût du risque ni a fortiori les charges de structure.

\section{Le coût de l'accompagnement}

Aux coûts portés par le prêteur s'ajoutent ceux portés par la CDC (appel en garantie, traitement administratif) et par les associations partenaires. La loi de 2010 spécifie en effet la nécessité d'un accompagnement social pour les emprunteurs. De façon concrète, cet accompagnement est essentiellement réalisé par les partenaires associatifs des prêteurs. Dans la majorité des réseaux distributeurs, la détection du besoin et la constitution du dossier de prêt sont réalisés, au sein des associations, par des employés ou des bénévoles souvent formés par le réseau prêteur. Pour les travailleurs sociaux, le MCP est un dispositif d'aide parmi d'autres. Y faire appel est un choix impliquant pour les structures sociales intermédiaires. Elles doivent, d'une part, affecter du temps à la formation de leurs employés ou des bénévoles pour qu'ils soient aptes à proposer ce produit dans les circonstances appropriées et, d'autre part, affecter du temps d'accompagnement dédié au MCP aux bénéficiaires de leur association. Des freins à la distribution peuvent ici apparaître à plusieurs titres. Les référents sociaux peuvent se sentir insuffisamment formés pour détecter le besoin, instruire le dossier, accompagner l'emprunteur. Les personnels des associations sont majoritairement polyvalents et peu de structures sont en capacité d'affecter un personnel dédié et expert sur ce sujet. Les référents sociaux peuvent aussi avoir développé une réticence à endetter les bénéficiaires, déjà précaires, ou encore arbitrer leur affectation du temps en défaveur du MCP et au profit d'autres actions d'accompagnement social. Par ailleurs, l'éclatement des distributeurs (six cents recensés en 2012 pour le réseau des Caisses d'épargne) ne favorise pas l'homogénéité des traitements, l'automatisation des tâches ou la professionnalisation de l'activité. L'enquête menée en 2012 par l'Union nationale des centres communaux d'action sociale (Unccas) auprès de 116 centres communaux d'action sociale (CCAS) et de 2 centres intercommunaux d'action sociale (CIAS) confirme ces problématiques. D’une part, elle souligne que le temps à dédier à l'activité MCP est important au regard des résultats obtenus. A l'issue du processus de sélection, seules $20 \%$ des demandes adressées aux CCAS aboutissent en effet à un prêt (Unccas, 2013). D’autre part, elle constate que les personnels investis sur le MCP peinent à développer cette activité. Parmi les CCAS interrogés, $72 \%$ aimeraient développer davantage le MCP, mais souhaiteraient pour cela que le dispositif soit amélioré par une meilleure collaboration avec d'autres acteurs locaux ( $42 \%)$, une révision des règles d'octroi au niveau national ( $41 \%$ ) et davantage de moyens alloués au sein de leur structure ( $28 \%)$. 
Ainsi, malgré la volonté politique - marquée tant par la législation que par l'effort financier consenti au titre des garanties accordées par la CDC d'accompagner la progression de la distribution du MCP, de nombreuses contraintes en limitent l'offre:

- contraintes de coût, pour les prêteurs;

- contraintes organisationnelles, pour les partenaires sociaux;

- contraintes de temps, pour les emprunteurs dont le besoin financier peut être immédiat et difficilement compatible avec les délais déclarés de déblocage des fonds.

Les caractéristiques actuelles du MCP (montant, taux, durée, sinistralité...) comme ses conditions de distribution (multitude de prêteurs, multitude d'intermédiaires, manque d'homogénéité des pratiques et d'automatisation...) ne sont donc pas favorables à son développement à grande échelle.

\section{Des arguments à renforcer pour justifier la dépense publique}

Pourtant, le microcrédit personnel garanti semble correspondre à un vrai besoin. L'Unccas (2013, p. 26) relève que « $41 \%$ des CCAS ressentent l'émergence de nouveaux publics demandeurs de microcrédit, notamment nouvellement exclus du système bancaire... L'arrivée de travailleurs pauvres parmi les demandeurs d'un prêt, ainsi que de retraités, se fait apparemment ressentir ». Toutefois, en l'état des choses, il est peu probable que les structures marchandes agissent en faveur de son développement. Parmi les structures sociales interrogées par l'Unccas en 2012,16 \% constatent même " une hausse des refus de MCP par la banque du fait des capacités financières insuffisantes des demandeurs».

\section{Les enseignements des études d'impact}

Aux réticences observées dans les sphères privées et associatives s'ajoutent des freins au développement des MCP garantis également observables dans la sphère publique. Les dépenses engagées doivent en effet pouvoir être justifiées par des améliorations sociales tangibles. Fin 2013, les études d'impact publiées concernent des emprunteurs issus de différents réseaux distributeurs, mais ont en commun l'objectif de mesurer la contribution du MCP à l'amélioration de la situation sociale et professionnelle des bénéficiaires. Quatre dimensions y sont principalement étudiées : l'amélioration de la situation professionnelle, de la situation budgétaire, du logement et de l'état moral de la personne. Les impacts mesurés peuvent varier significativement selon l'enquête source. Le MCP apparaît toutefois comme une solution d'aide opportune, bien que perfectible.

Le tableau 6 (page suivante) montre que les taux de satisfaction déclarée calculés dans les quatre enquêtes citées et sur les quatre principaux axes d'amélioration des conditions de vie peuvent encore progresser. Ainsi, outre le fait qu'il soit coûteux pour le prêteur, complexe à organiser et chronophage pour les intermédiaires, le MCP n'atteint pas toujours son but d'amélioration du quotidien pour les emprunteurs. 


\section{Tableau 6}

\section{Impacts du microcrédit personnel (2010-2013)}

\begin{tabular}{|c|c|c|c|c|}
\hline & $\begin{array}{l}\text { Fédération } \\
\text { nationale } \\
\text { des Caisses } \\
\text { d'épargne } \\
\text { et CDC }\end{array}$ & $\begin{array}{l}\text { Crédit } \\
\text { municipal } \\
\text { de Paris } \\
\text { (Aldeghi, Olm, } \\
2001 \text { ) }\end{array}$ & $\begin{array}{l}\text { Adie } \\
\text { (rapport annuel } \\
\text { de l'Observatoire } \\
\text { de la micro- } \\
\text { finance 2011) }\end{array}$ & $\begin{array}{l}\text { Cosef - CDC } \\
\text { (Gloukoviezoff, } \\
\text { Rebière, 2013b) }\end{array}$ \\
\hline Année de l'étude & 2010 & 2011 & 2011-2012 & 2012-2013 \\
\hline Panel d'emprunteurs & 807 & 300 & 1400 & 1495 \\
\hline \multicolumn{5}{|l|}{$\begin{array}{l}\text { Pourcentage } \\
\text { de répondants } \\
\text { ayant perçu } \\
\text { une amélioration sur: }\end{array}$} \\
\hline •l'emploi & $\begin{array}{r}80 \% \\
\text { des répondants }\end{array}$ & $33 \% *$ & $\begin{array}{l}\text { Objectifs } \\
\text { atteints par: } \\
51 \% \text { des clients } \\
\text { visant le retour } \\
\text { à l'emploi et } 83 \% \\
\text { des clients visant } \\
\text { le maintien } \\
\text { dans l'emploi }\end{array}$ & $51 \% *$ \\
\hline $\begin{array}{l}\text { - la situation } \\
\text { budgétaire }\end{array}$ & $57 \%$ & $55 \%$ & NC & $28 \%$ \\
\hline - le logement & $68 \% * *$ & $48 \% * *$ & & $63 \% * *$ \\
\hline $\begin{array}{l}\text { - le plan moral, l'estime } \\
\text { de soi }\end{array}$ & $58 \%$ & $35 \%$ & & $45 \%$ \\
\hline
\end{tabular}

Source : synthèse après analyse de rapports d'études publiés entre 2010 et 2013.

* Pourcentage des répondants qui ont financé un projet professionnel.

** Pourcentage des répondants qui ont financé un projet en lien avec le logement.

Tous projets et tous emprunteurs confondus, l'enquête 2013 de la CDC révèle des taux d'amélioration et de préservation cumulés systématiquement en deçà de 53 \% qui interrogent sur l'adéquation de l'aide apportée et donc sur la correcte affectation des fonds publics.

Ces résultats plaident pour la mise en œuvre d'une évaluation systématique de l'issue du MCP. Cette action présenterait un triple avantage : une meilleure visibilité pour légitimer l'affectation des fonds publics ; une meilleure analyse des causes de non-adéquation ; l'assurance du suivi requis par réglementation, mais peu réalisé en pratique. Du fait du temps qu'il requiert pour les associations ou des réticences des emprunteurs à être davantage encadrés, au moins un bénéficiaire de MCP sur deux n'est en effet plus suivi après le déblocage des fonds ${ }^{(6)}$. Or, la difficulté financière n'est bien souvent qu'une conséquence de difficultés sociales de plus grande ampleur : professionnelles, comportementales, familiales ou encore en lien avec la santé du bénéficiaire. Ces difficultés, pour être amoindries, requièrent bien une prise en charge globale de la personne. Le MCP n'intervient qu'en complément dans un processus censé améliorer l'autonomie et la capacité à se projeter du bénéficiaire.

(6) «A la question de savoir si, en dehors de tout incident depaiement de l'emprunteur, les CCAS ont mis en place des actions de suivi ou d'accompagnement des bénéficiaires (ateliers budget ou entretiens de suivi proposés auxemprunteurs parfois à la demande du partenaire bancaire), ils sont près d'un tiers à répondre "non", un tiers "oui, parfois" et un tiers "oui, systématiquement” (Unccas, 2013). 


\section{Tableau 7}

Etude CDC 2013 : impacts du microcrédit personnel garanti

Améliore

Préserve

Neutre

Négatif

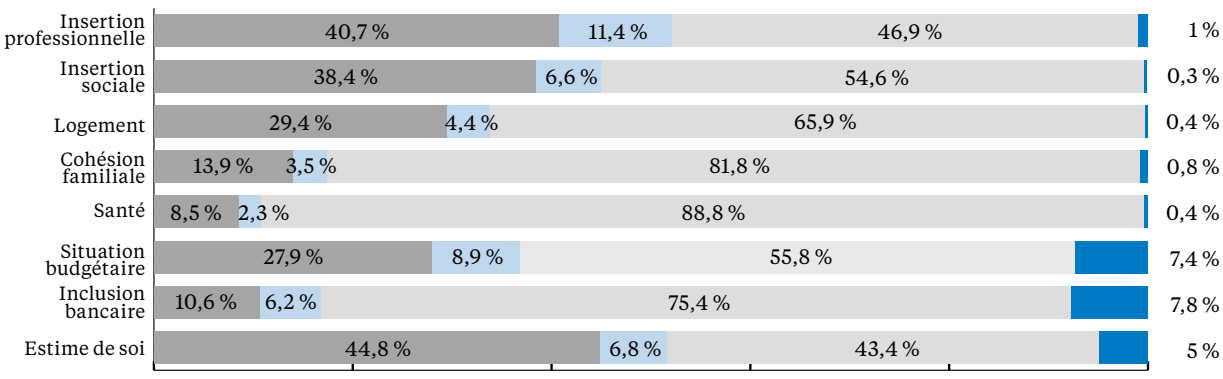

Source : Gloukoviezoff, Rebière (2013b, livret 5).

Contrairement au microcrédit professionnel, focalisé sur l'emploi, l'enjeu du microcrédit personnel est avant tout l'amélioration des conditions de vie. Ce produit, comme les aides sociales, est un instrument d'inclusion. Ces caractéristiques (modalités de diffusion, implication des pouvoirs publics) l'assimilent d'ailleurs davantage à celles-ci qu'à un véritable produit bancaire porté par une logique purement marchande. De ce fait, l'attribution d'un MCP doit porter en elle la responsabilité d'un accompagnement global. Le MCP doit avoir la vertu de démontrer qu'un projet construit, anticipé, suivi jusqu'à son terme peut améliorer les conditions de vie. Après attribution, il serait cohérent d'aller au bout de cette démarche formatrice et d'évaluer systématiquement l'issue du projet, tant pour limiter le coût du risque que pour accentuer les effets individuels positifs attendus des MCP.

A titre d'illustration, il est intéressant de reprendre la statistique suivante, observée dans le rapport FNCE (2010) : "Quasiment un véhicule sur deux acheté grâce au microcrédit fait l'objet d'une réparation dans les deux ans, dont près d'un tiers dans les six mois. Un événement susceptible de compromettre la réalisation du projet et le remboursement du microcrédit s'il n'a pas été anticipé en amont. » Dans cette étude, $65 \%$ des projets financés étaient axés sur la mobilité. Au 31 décembre 2012, les statistiques de la CDC font apparaître que l'emploi et la mobilité sont l'objet de 73 \% des prêts. Ces chiffres montrent bien que l'accès au financement n'est pas une fin en soi, mais un moyen dans le cadre d'une démarche d'autonomisation plus globale.

Plusieurs facteurs peuvent expliquer l'absence de suivi systématique réalisé aujourd'hui : le temps à y affecter, les difficultés à mobiliser l'emprunteur après le déblocage des fonds, le manque de moyens (financiers ou pour la formation de l'accompagnateur) pour apporter des solutions alternatives si le projet initial connaît des difficultés sont les causes les plus fréquemment citées par les structures partenaires des établissements financiers prêteurs. 
Selon l'angle de lecture des études d'impact réalisées, il peut encore être difficile pour l'Etat de justifier les dépenses affectées au MCP et donc d'en promouvoir une diffusion plus large. Un renforcement de l'évaluation des effets sociaux des MCP accordés pourrait limiter cet obstacle.

\section{Un objet flou, une dépense publique utile?}

Il ne suffirait pas toutefois à assurer l'entière légitimité du dispositif. Sa conception même en contraint en effet son développement. Contrairement au microcrédit professionnel, l'objet du microcrédit personnel est relativement large. La loi prévoit de garantir par le Fonds de cohésion sociale les "prêts destinés à participer au financement de projets d'insertion... accordés afin de permettre l'accès, le maintien ou le retour à un emploi. Ces prêts peuvent également être accordés pour la réalisation de projets d'insertion sociale qui ne sont pas directement liés à un objectif professionnel ${ }^{(7)}$. En théorie, le financement peut donc être affecté à des objets très divers, en fonction de la situation du demandeur. Dans la pratique, les résultats d'enquêtes montrent qu'environ $70 \%$ des prêts sont destinés à améliorer la mobilité de l'emprunteur. Cependant, les

(7) Loi no 2005-32 du 18 janvier 2005 de programmation pour la cohésion sociale, article 80, III. analyses révèlent également une "affectation "trésorerie domestique" importante. Elle concerne à la fois des paiements d'arriérés de factures, des petits crédits en cours, de la couverture de découverts bancaires autorisés pour soi ou pour ses proches, des combinaisons "mobilité et trésorerie domestique" ", notamment (Glémain, 2012). En 2010 déjà, une étude locale soulignait que près d'un tiers des crédits servaient à financer "les fins de mois difficiles ou à rembourser les mensualités d'un crédit revolving à la marge des exigences du Plan de cohésion sociale qui interdit tout financement de trésorerie» (Moulévrier, 2012). Pour certains auteurs, «la richesse du dispositif de microcrédit personnel tient précisément à sa facultéà répondre à une grande variété de demandes de financement » (Gloukoviezoff, Rebière, 2013a).

Cependant, la diversité des objets financés par le MCP peut être perçue comme un frein à l'expansion du dispositif, car elle rend plus difficile la justification de la dépense publique. En tout état de cause, cette dernière peut-elle être mobilisée pour financer des décalages de trésorerie ou pour favoriser l'endettement non productif d'emprunteurs exclus du système bancaire traditionnel?

La "solidarité collective ne saurait financer les dépenses futiles de personnes qui n'en ont pas les moyens » (Gloukoviezoff, Rebière, 2013a). La question de la légitimité de l'objet financé renvoie aux critiques faites à Amartya Sen, prix Nobel d'économie 1998, sur l'opérationnalité de l'approche par les capacités (Farvaque, 2008). La liberté relative dans l'affectation du financement garanti peut élargir les opportunités de l'emprunteur - capacité de choix - et son potentiel d'épanouissement. L'accompagnement associé au financement peut faciliter la conversion de ce potentiel en accomplissement. Le microcrédit personnel garanti peut donc apparaître comme un vecteur de "capacité» telle que redéfinie par De Munck (2008) à la lecture de Sen, mais ce dernier n'établit pas de liste des capacités fondamentales et ne se prononce pas sur la légitimité des pouvoirs publics à intervenir sur les capacités les moins urgentes (Ogien, 2008). Ainsi, les références manquent pour trancher le débat de la dépense légitime. 


\section{Conclusion}

Le bilan du MCP apparaît très mitigé: il n'atteint qu'une faible proportion de la population pour laquelle il a été conçu, il présente des effets inégaux sur les emprunteurs, les taux d'amélioration de la situation des emprunteurs calculés sur divers critères dépassant rarement $40 \%$, il aboutit à une impasse économique pour les établissements prêteurs et, chronophage, il réclame des arbitrages aux associations dont les ressources tant humaines que financières sont limitées. Economiquement, d'après ses caractéristiques intrinsèques, le microcrédit personnel garanti n'est pas viable sans aide massive des pouvoirs publics. Il n'est pas un produit financier, mais un dispositif social. Or, au-delà des limites matérielles, il présente aussi par construction des freins conceptuels, puisque ni les limites d'intervention des pouvoirs publics ni ses objectifs - spécifiques, mesurables, acceptables par les différentes parties prenantes, réalisables et temporels - ne sont précisément définis.

Ces constats renvoient donc à un double questionnement. Cette activité doitelle entrer dans le secteur marchand et devenir économiquement soutenable, par une hausse des taux pratiqués et une automatisation des process? En complément ou intégralement, dans quelle mesure et pour quels résultats cette activité doit-elle être financée par les pouvoirs publics? Pour répondre à ces questions, il peut être utile de rappeler les règles de Tinbergen (1956) et de Mundell (1960). La première énonce que, pour toute politique économique ayant des objectifs fixés, le nombre d'instruments doit être égal au nombre d'objectifs visés, et la seconde implique d'affecter l'instrument le plus efficace à l'objectif. Concernant le MCP, à la fois les objectifs et les instruments (crédit, accompagnement, évaluation...) restent flous, ce qui contribue à la sous-optimalité du système en vigueur.

Aussi longtemps que les freins tant économiques que conceptuels ne seront pas levés, il semble peu probable que le développement du microcrédit personnel aille au-delà du discours d'intention. Raisonnablement couteux à titre expérimental, il laisse croire en l'existence d'un dispositif à visée inclusive généralisé à défaut de proposer une ou des actions de fond pour répondre plus largement aux besoins diversifiés auxquels il peut correspondre. Les objectifs et les instruments du MCP doivent donc être précisés pour faire de ce dispositif un facteur d'inclusion efficace. 


\section{BIBLIOGRAPHIE}

Aldeghi I., Olm C., 2011, " Impact socioéconomique du microcrédit mis en place par le Crédit municipal de Paris sur la situation des emprunteurs ", rapport réalisé par le Crédoc pour le Crédit municipal de Paris, juillet 2011.

Autorité de contrôle prudentiel, 2012, "Enquête annuelle sur le financement de l'habitat en 2011 ", Analyses et Synthèses, $\mathrm{n}^{\circ} 8$, Banque de France.

Banque de France, 2012, rapport annuel de l'Observatoire de la microfinance, exercice 2011.

De Munck J., 2008, « La liberté au prisme des capacités: Amartya Sen au-delà du libéralisme ", Qu'est-ce qu'une capacité? Editions de 1'Ecole des hautes études en sciences sociales.

Ducourant H., 2010, « L’offre de crédit dans les catalogues de vente par correspondance 1974-2001: de l'outil de gestion budgétaire à l'accompagnement des ménages dans leur carrière de consommateurs", Entreprises et Histoire, $\mathrm{n}^{\circ}$ 59, p. 41-56.

Farvaque N., 2008, "La liberté au prisme des capacités: Amartya Sen au-delà du libéralisme ", Faire surgir des faits utilisables, Editions de l'Ecole des hautes études en sciences sociales.

Fédération nationale des Caisses d'épargne, 2010, Etude d'impact du microcrédit personnel: évolution de la situation des emprunteurs.

Fédération nationale des Caisses d'épargne, 2013, rapport annuel RSE des Caisses d'épargne 2012.

Glémain P., dir., 2012, Collectif de recherches finances et monnaies solidaires, "Le crédit personnel garanti: une analyse transdisciplinaire de l'accompagnement dans le cadre d'un service bancaire solidaire", rapport final à la direction générale à la cohésion sociale, ministère délégué à l'Economie sociale et solidaire.
Glémain P., 2012, "Le microcrédit personnel garanti "à la nantaise" ", Collectif de recherche finances et monnaies solidaires (Fimosol), colloque du 20 janvier 2012.

Glemain P., Meyer M., 2011, « Aide sociale et/ou action sociale? De la philosophie du microcrédit personnel garanti et de ses acteurs ", Politique et Management public, vol. 28, n 3 , p. 261-78.

Glémain P., Moulévrier P., 2011, « Le microcrédit: un crédit comme les autres?", La revue des sciences de gestion, $\mathrm{n}^{\text {os }} 249-250$, p. 123-131.

Gloukoviezoff G., Rebière N., 2013a, Microcrédits contre pauvreté: des prêts entre solidarité et marché, Les éditions de l'Atelier, Paris.

Gloukoviezoff G., Rebière N., 2013b, «Evaluation d'impacts du microcrédit personnel garanti », rapport pour la Caisse des dépôts et consignations.

Inspection générale des finances, 2010, Le microcrédit, rapport $n^{\circ}$ 2009-M-085-03, ministère de l'Economie, de l'Industrie et de l'Emploi.

Moulévrier P., 2012, «Les structures sociales du marché bancaire en France", Revue française de socioéconomie, 2012, vol. 1, n 9 , p. 23-41.

Ogien A., 2008, «La liberté au prisme des capacités: Amartya Sen au-delà du libéralisme ", Arithmétique de la liberté: la mesure des capacités et ses paradoxes, Editions de l'Ecole des hautes études en sciences sociales. Mundell R. A., 1960, « The monetary dynamics of international adjustment under fixed and flexible exchange rates ", The Quarterly Journal of Economics, p. 227-257.

Tinbergen J., 1956, Economic Policy: Principles and Design, North Holland.

Unccas, 2013, "Les modalités de mise en place du microcrédit personnel: étude des disparités entre CCAS et CIAS », rapport d'enquête. 
A RETROUVER DANS LES ARCHIVES DE LA RECMA (RECMA.ORG/ARTICLES)

Lapenu C., Doligez F., "Mesure des performances sociales: les implications pour le secteur de la microfinance », Recma, $\mathrm{n}^{\circ}$ 304, 2007.

Guérin I., Servet J.-M., "L'économie solidaire entre le local et le global: l'exemple de la microfinance », Recma, $\mathrm{n}^{\circ}$ 296, 2005.

Tchouassi G., Tekam Oumbe H., « Microfinance et réduction de la pauvreté: le cas du Crédit du Sahel au Cameroun », Recma, n² 288, 2003.

Malo M.-C., Lapoutte A., "Caisse d'épargne et Adie: une configuration partenariale innovatrice ", Recma, $\mathrm{n}^{\circ}$ 286, 2002. 